\title{
Two-path transport measurements on a triple quantum dot
}

\author{
M. C. Rogge* and R. J. Haug \\ Institut für Festkörperphysik, Leibniz Universität Hannover, Appelstrasse 2, 30167 Hannover, Germany
}

(Received 23 October 2007; published 8 May 2008)

\begin{abstract}
As an advanced quantum system, a three terminal triple quantum dot with one lead attached to each dot allows us to simultaneously measure a transport along two different paths. Quadruple points with all three dots in resonance are prepared and investigated for different electron numbers in the individual quantum dots including one dot with only one or two electrons.
\end{abstract}

DOI: 10.1103/PhysRevB.77.193306

PACS number(s): 73.21.La, 73.23.Hk, 73.63.Kv

The spin of electrons on a quantum dot is proposed as a realization of a qubit that is needed for quantum computation. ${ }^{1}$ Therefore, quantum dots, the so-called artificial atoms, have been intensively investigated in recent years. ${ }^{2}$ Next to single quantum dots, two coupled quantum dots (artificial molecules) have gained broad interest. ${ }^{3}$ Coupling phenomena have been investigated in devices with parallel $^{4,5}$ as well as with serial ${ }^{6-9}$ configurations. The ongoing technological progress has allowed the fabrication of more and more complicated setups, including charge detection with quantum point contacts (QPCs). ${ }^{10,11}$ Recently, the technological and scientific preconditions have reached a level that allows one to go beyond single and coupled qubits. An exciting field of research is being entered: the investigation of triple quantum dots.

Next to fundamental physics, the research on triple quantum dots is motivated by the fact that a triple quantum dot is the smallest system, with quantum dots being part of a qubit chain. However, despite this importance for quantum computation and further interesting theoretical predictions, ${ }^{12-15}$ triple quantum dots have almost not been investigated so far (except for some basic experiments in the early $1990 \mathrm{~s}^{16,17}$ ) due to experimental difficulties. Now that researchers have started to overcome these difficulties, the intensive investigation of triple dots has begun with three recently published experiments. Vidan et al. ${ }^{18}$ investigated a serial double quantum dot with a side coupled third dot as a quantum box. Gaudreau et al. ${ }^{19}$ observed charge rearrangements on a ringlike triple quantum dot in a system that was originally designed for double quantum dots. Schröer et al. ${ }^{20}$ created a system with three dots in a row.

In this Brief Report, we present a different geometry for a lateral triple quantum dot. We created a starlike system with each dot placed next to the other two. In contrast to the formerly published works, we have three leads connected to our system, one for each quantum dot. Thus, we can simultaneously measure the transport via different paths with only two dots per path. With this setup, transitions from configurations of two dots in resonance (triple points) to configurations of all three dots in resonance (quadruple points) can be studied in transport in order to understand the formation of triple quantum dot states.

To enable charge detection, we extend our device by a QPC, making the setup more flexible. With this variety of features, we decided to use an atomic force microscope (AFM) to build this unique setup, as this technique provides the same functionality with fewer gates involved compared to devices made by split gate technique with e-beam lithography. Therefore, as far as we know, this is the only lateral triple quantum dot made with local anodic oxidation (LAO). ${ }^{21,22}$

Using LAO on a GaAs/AlGaAs heterostructure, oxide lines are created, which are shown in black in the AFM image of the device in Fig. 1 (left hand side). Three dots (A, B, and C) (see dashed circles) are defined in a starlike setup with tunneling barriers in between. Each dot is connected to a "personal" lead used as the source or drain contacts. Four gates $\left(G_{1}-G_{4}\right)$ are used to tune the coupling to the leads and the interdot coupling. Due to the small dimensions, the gates do not have an independent influence and thus a fine balance of all four gate voltages is necessary to operate the system. For a charge detection, a QPC (dashed lines) is placed below dots $\mathrm{B}$ and $\mathrm{C}$ with its own source and drain leads ( source $_{\mathrm{QPC}}$ and drain $_{\mathrm{QPC}}$ ) and an additional gate $G_{\mathrm{QPC}}$ to tune the conductance of the QPC. The complete QPC can be used as another gate $\left(G_{5}\right)$ for the triple dot with the charge detection still working.
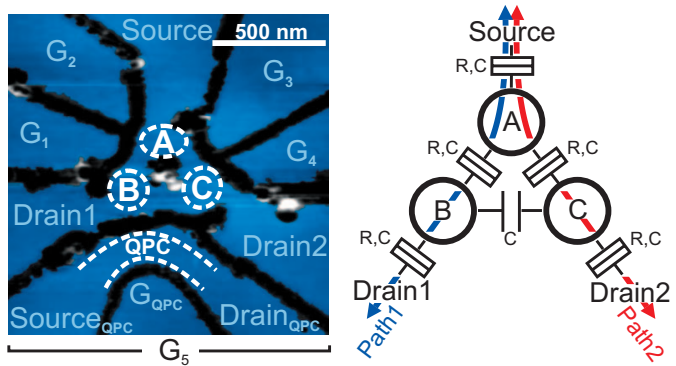

FIG. 1. (Color) Left: colored AFM image of our triple dot device defined by oxide lines (dark). Three quantum dots (A, B, and C) are placed in the center of the device, such that each dot is connected via tunneling barriers to the other two. Each dot has its own lead which can serve as a source or drain contact. The displayed setup with source at $\operatorname{dot} \mathrm{A}$, drain 1 at $\mathrm{B}$, and drain 2 at $\mathrm{C}$ is used for two-path transport measurements. Four gates $\left(G_{1}-G_{5}\right)$ control the potentials of the dots and the barriers. A QPC is placed next to dots $\mathrm{B}$ and $\mathrm{C}$ for charge detection. It has its own source and drain leads (source $\mathrm{QPC}_{\mathrm{QPC}}$, drain $\mathrm{QPC}_{\mathrm{QPC}}$ ) and a tuning gate $\mathrm{G}_{\mathrm{QPC}}$. The complete QPC can be used as an additional gate $\left(\mathrm{G}_{5}\right)$ with the QPC still working. Right: schematic for the triple dot setup. The three dots are coupled via tunneling barriers $(R, C)$ to the leads and to each other; dots B and C are capacitively coupled only (C). Transport is measured via two paths, along dots A and B (path 1) and A and $\mathrm{C}$ (path 2). 

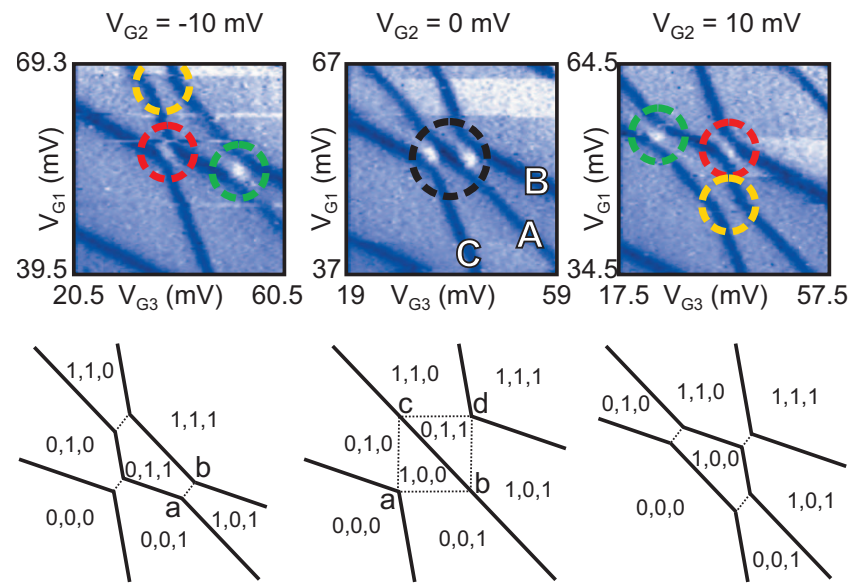

FIG. 2. (Color) Charge detection of triple dot states. The differentiated current through the QPC $d I_{\mathrm{QPC}} / d V_{G_{3}}$ is plotted as a function of the two gate voltages for different values of $V_{G_{2}}$ with $V_{G_{4}}$ $=0$. In each image, dark features are visible with different slopes. They denote states from all three dots (marked for $V_{G_{2}}=0 \mathrm{mV}$ ). Anticrossings appear for resonances of two of the three dots (marked with circles; red for resonances of $\mathrm{B}$ and $\mathrm{C}$, green for $\mathrm{B}$ and $\mathrm{A}$, and yellow for $\mathrm{C}$ and $\mathrm{A}$ ). With $V_{G_{2}}$, those can be shifted to establish a triple dot resonance (black circle for $V_{G_{2}}=0 \mathrm{mV}$ ). The simplified schematics below show the corresponding electronic configurations. The numbers count the electrons added to dots A, B, and $\mathrm{C}$ relative to the configuration at the lower left.

The measurements were performed in a ${ }^{3} \mathrm{He} /{ }^{4} \mathrm{He}$-dilution refrigerator at a base temperature of $15 \mathrm{mK}$. ac and dc voltages were applied to one lead of the triple dot called the source, while the differential conductance $G$ through the device was measured on the other two leads called drain 1 and drain2, each of which uses two lock-in setups. The QPC was operated with a dc voltage applied to source $_{\mathrm{QPC}}$ and with a dc measured at drain ${ }_{\mathrm{QPC}}$. In the following, the device is connected, as shown in Fig. 1: the source contact is connected to $\operatorname{dot} \mathrm{A}$, drain 1 is placed at $\operatorname{dot} \mathrm{B}$, and drain 2 at $\operatorname{dot} \mathrm{C}$. Thus,

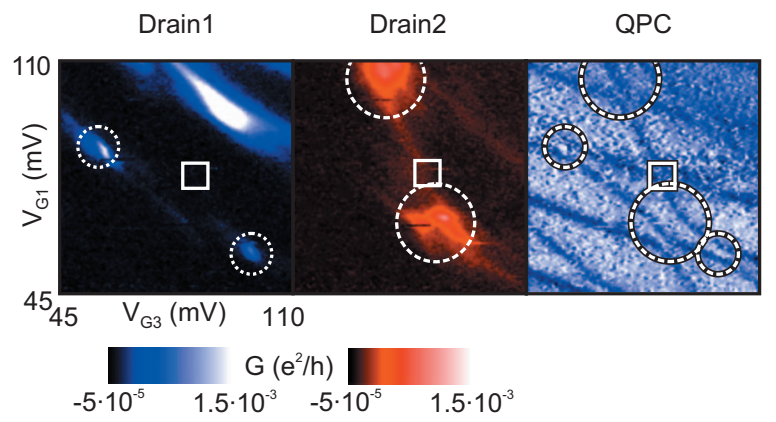

FIG. 3. (Color) Differential conductance $G$ measured at drain1 (blue) and drain2 (red) with the corresponding charge measurement as a function of $V_{G_{3}}$ and $V_{G_{1}}$ with $G_{4}$ at $-100 \mathrm{mV}$. Along both paths, spots of finite $G$ show serial transport due to resonances of the corresponding two dots (marked with small circles along path 1 and big circles along path 2). These spots correspond to anticrossings as shown in the QPC measurement. Blue spots denote resonances of dots $\mathrm{A}$ and $\mathrm{B}$; red spots appear for resonances of dots $\mathrm{A}$ and $\mathrm{C}$. The QPC measurement shows further resonances of dots B and $\mathrm{C}$ (square). Those are not visible in transport.

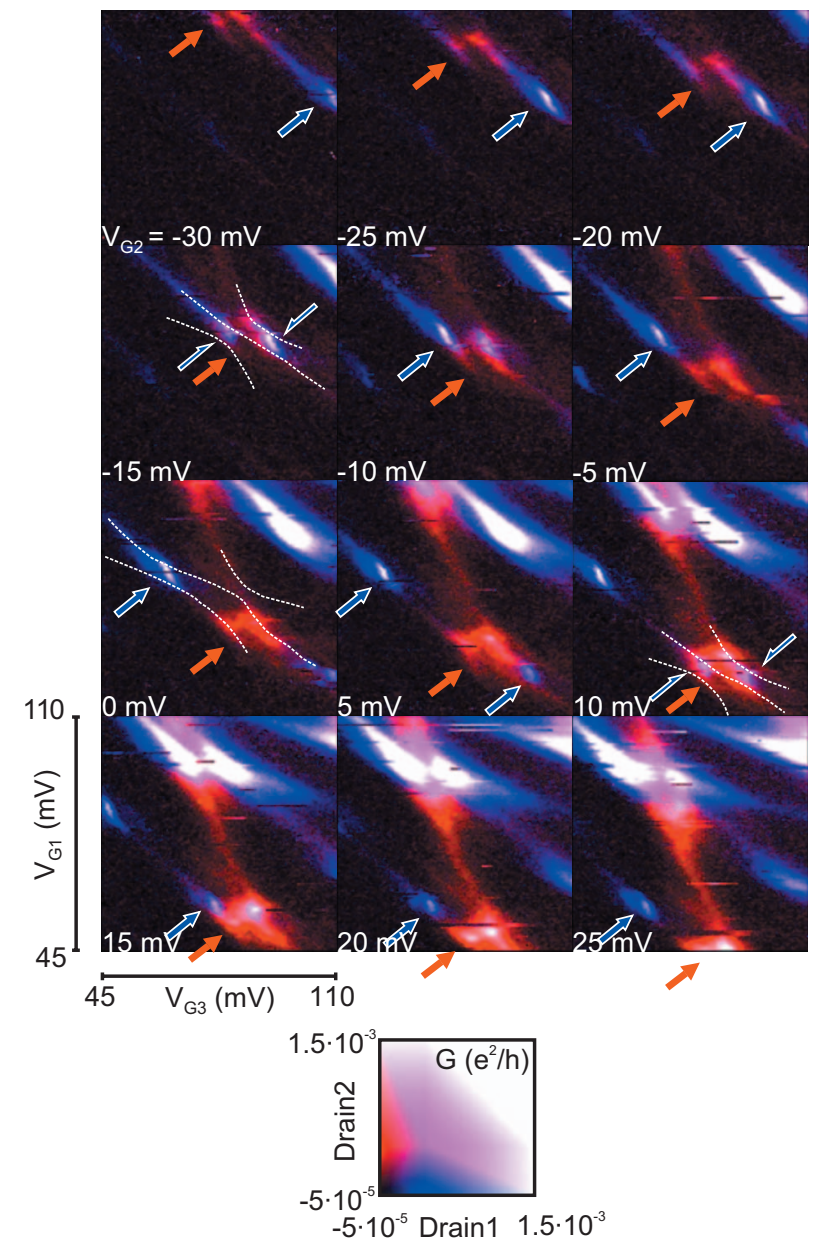

FIG. 4. (Color) Differential conductance $G$ along both paths (along dots $\mathrm{A}$ and $\mathrm{C}$ in red, along dots $\mathrm{A}$ and $\mathrm{B}$ in blue) as a function of $V_{G_{3}}$ and $V_{G_{1}}$ while stepping $V_{G_{2}}$ from -30 (upper left) to $25 \mathrm{mV}$ (lower right). $V_{G_{4}}$ is set at $-100 \mathrm{mV}$. $V_{G_{2}}=5 \mathrm{mV}$ is identical to the measurement shown in Fig. 3. Along both paths, spots of finite $G$ show serial transport due to resonances of the corresponding two dots, some of them marked with accordingly colored arrows. With $V_{G_{2}}$, those are shifted and triple resonances are established. Those appear for $V_{G_{2}}=-15 \mathrm{mV}$ and $V_{G_{2}}=10 \mathrm{mV}$. Further triple resonances are found for higher voltages (e.g., for $V_{G_{2}}=20 \mathrm{mV}$ ). White dashed lines show the charging lines measured with the QPC.

transport can be measured in two parallel paths, as shown in the schematic on the right hand side of Fig. 1, with dots A and $\mathrm{B}$ in series along path 1 and dots $\mathrm{A}$ and $\mathrm{C}$ in series along path 2 .

First, the device is studied in the closed regime where no current is detectable along both paths. Still the charging of the device is detectable using the QPC. This is shown in Fig. 2. The derivative of the QPC current is plotted as a function of gates $G_{3}$ and $G_{1}$ for three different voltages at gate $G_{2}$. In each measurement, dark lines that denote charging events in the system are visible. As the slopes of these lines depend on the distance between a dot and the two gates, three different slopes are visible, one for each dot. The lines with the lowest slope belong to dot B; those with the largest slope belong to $\operatorname{dot} \mathrm{C}$. The lines with intermediate slopes denote charging on $\operatorname{dot} \mathrm{A}$ (see Fig. 2, $V_{G_{2}}=0 \mathrm{mV}$ ). Anticrossings are found with 
two dots in resonance where the lines meet. In Fig. 2 at $V_{G_{2}}=-10 \mathrm{mV}$, those anticrossings are visible for resonance between dot $\mathrm{A}$ and dot $\mathrm{B}$ (green circle, the chemical potentials for dot $\mathrm{A}$ and dot $\mathrm{B}$ are equal, $\mu_{\mathrm{NA}}=\mu_{\mathrm{NB}}$ with NA and $\mathrm{NB}$ as the electron numbers on both dots), dot $\mathrm{A}$ and dot $\mathrm{C}$ (yellow circle, $\mu_{\mathrm{NA}}=\mu_{\mathrm{NC}}$ ), and dot $\mathrm{B}$ and dot $\mathrm{C}$ (red circle, $\left.\mu_{\mathrm{NB}}=\mu_{\mathrm{NC}}\right)$. The anticrossings between dots $\mathrm{A}$ and $\mathrm{B}$ and between dots $\mathrm{A}$ and $\mathrm{C}$ appear due to capacitive and tunneling coupling. Although no tunnel coupling is possible between B and $\mathrm{C},{ }^{23}$ there is still a huge capacitive coupling, which demonstrates the close vicinity of the two dots. At these anticrossings, bright features are visible due to charge transitions from one dot to another without changing the total charge of the system. For $V_{G_{2}}=-10 \mathrm{mV}$ in Fig. 2, all three anticrossings are separated by a few millivolts. For $V_{G_{2}}=0 \mathrm{mV}$, these anticrossings coincide (black circle), thus showing the resonance condition for all three dots $\left(\mu_{\mathrm{NA}}=\mu_{\mathrm{NB}}=\mu_{\mathrm{NC}}\right)$. With increasing $V_{G_{2}}$, the resonances are shifted further and the resonance condition for three dots is lifted again $\left(V_{G_{2}}\right.$ $=10 \mathrm{mV}$ ). Thus, the existence of three coupled quantum dots with tunable resonance conditions is demonstrated. Furthermore, dot $\mathrm{C}$ can be emptied to zero electron as the line visible for dot $\mathrm{C}$ is the last line detected. No further line corresponding to $\mathrm{C}$ appears when decreasing the gate voltages. The line visible denotes charging with the first electron $\left(\mu_{\mathrm{NC}}=\mu_{1 \mathrm{C}}\right)$.

The schematics at the bottom of Fig. 2 show the corresponding electronic configurations ranging from $0,0,0$ to $1,1,1$. Off triple dot resonance (left and right schematics), either the configurations $0,1,1$ or 1,0,0 appear in the middle. On resonance (central schematic), both configurations are visible. The typical triple points that connect three configurations in double dot systems ( $\mathrm{a}$ and $\mathrm{b}$ at left schematic) are replaced by four quadruple points on resonance for the triple dot system (a, b, c, and d at central schematic). They connect four configurations. At these points, transport can occur. At quadruple point a, an electronlike transport is possible along both paths, while $\mathrm{d}$ features a holelike transport along both paths. At $b$, electrons can pass the device via drain 1 and holes via drain2, and at c, vice versa.

Indeed, adjusting the gate voltages and opening the barriers, finite transport through the dots becomes measurable. This is shown in Fig. 3. Charge diagrams are recorded sweeping at gates $G_{3}$ and $G_{1}$, as in Fig. 2 . Next to the charge detection, the differential conductance $G$ is measured along paths 1 and 2. The measurement along path 1 is plotted in blue and the one along path 2 in red. Along both paths, spotlike features are visible, some of them marked with circles. Comparing these features with the QPC measurement, one finds that they correspond to anticrossings of quantum dot states. The spots measured at drain1 (small circles) correspond to resonances between dots A and B. The features measured at drain2 (big circles) appear due to resonances between dots $\mathrm{A}$ and $\mathrm{C}$. The latter ones are slightly split due to the strong interdot coupling of $\mathrm{A}$ and $\mathrm{C}$. Thus, transport occurs at the two triple points on both sides of the anticrossing. Due to the smaller distance of the anticrossing for dots A and B, the two triple points merge in transport and drain 1 shows only single spots with a maximum in the center of the anticrossing. In both paths, at least two spots are visible, which means that these are resonances for different electron numbers. The two marked blue spots appear for the same state on dot A but for two consecutive states on dot B. Thus, at one spot, dot B is charged with an even number of electrons and at the other resonance with an odd number. Similar properties account for the red features. Both spots appear for the same state on dot $\mathrm{C}$, which is charging here with the second electron (transport for the first electron can be measured for different gate voltages). Two consecutive states on dot A are involved, one of them with even and the other with odd electron numbers. The charge signal at the QPC shows a third group of anticrossings that are exemplarily marked with a square. Those correspond to resonances between dots $\mathrm{B}$ and $\mathrm{C}$. Comparing these features with the measurements along both paths, it becomes obvious that there are no corresponding features in transport. This remarkably shows the functionality of the two-path setup with a common source contact and two drain contacts. In each path, the two dots placed there must be in resonance to allow for serial transport. Resonances of two dots in different paths are not sufficient to generate finite differential conductance.

With the ability to measure conductance along two paths simultaneously but separately, the data can be analyzed in combined color scale plots, as shown in Fig. 4. Both signals are plotted together and are distinguishable by different colors (red for path 1 and blue for path 2). In this way, positions of features in both paths can be compared in detail. As in Fig. 2, the resonances visible in transport can be shifted to establish resonances of all three quantum dots. For each measurement shown in Fig. 4, $V_{G_{2}}$ is set at a fixed voltage starting from $-30 \mathrm{mV}$ at the upper left and is increased to $25 \mathrm{mV}$ at the lower right. Both paths show spotlike features due to the resonance of two dots (color encoded as in Fig. 3), some of them marked with blue and red arrows. While the red spots move downward with increasing $V_{G_{2}}$, the blue spots move to the left. Thus, using gate $G_{2}$ resonance conditions for all three dots can be created. The two spots marked with a red and a blue arrow for $V_{G_{2}}=-30 \mathrm{mV}$ (chemical potentials $\mu_{\mathrm{NA}}=\mu_{\mathrm{NB}}$ and $\mu_{\mathrm{NA}}=\mu_{2 \mathrm{C}}$ ), for example, approach each other with increasing $V_{G_{2}}$. At $V_{G_{2}}=-15 \mathrm{mV}$, both spots have merged, simultaneous transport via path 1 and path 2 is detected, and the three dots are in resonance $\left(\mu_{\mathrm{NA}}=\mu_{\mathrm{NB}}\right.$ $\left.=\mu_{2 \mathrm{C}}\right)$. The blue features appear at the transition from $0,1,1$ to $1,0,1$ and $0,1,0$ to $1,0,0$ (see schematics in Fig. 2). At resonance, both transitions appear separated by a few millivolts. Therefore, the blue spot is split into two spots connecting the quadruple points a,c and b,d. A further increase in $V_{G_{2}}$ moves the blue and red spots apart again. The same happens with the red spot and another blue spot coming into resonance at $V_{G_{2}}=10 \mathrm{mV}\left(\mu_{\mathrm{NA}}=\mu_{\mathrm{NB}-1}=\mu_{2 \mathrm{C}}\right)$. Off resonance, the red spots coincide with the charging lines detected with the QPC (white dashed lines), while blue spots appear in between (see $V_{G_{2}}=0 \mathrm{mV}$ ). On resonance, the red features follow the split blue spots and appear between the charging lines as well (see $V_{G_{2}}=-15 \mathrm{mV}$ and $V_{G_{2}}=10 \mathrm{mV}$ ). The two triple resonances differ by one electron on dot B. As mentioned before, further resonances are visible at higher $V_{G_{1}}$ $\left(\mu_{\mathrm{NA}+1}=\mu_{\mathrm{NB}+1}=\mu_{2 \mathrm{C}}\right.$ at $\left.V_{G_{2}}=20 \mathrm{mV}\right)$. Here, the electron 
number on dot A has changed as well. Similar results were gained by stepping $V_{G_{4}}$ instead of $V_{G_{2}}$.

Thus, not only with charge detection but even in transport can we detect clear resonances of two quantum dots in two different paths in combined color plots. Shifting these resonances, quadruple points can be formed with all three dots in resonance. These quadruple points can be prepared for different electron numbers creating odd or even configurations on each dot and, thus, on the whole triple dot as well. Therefore, this device is promising to verify theoretical predictions recently published for two-path triple quantum dots. A twopath triple dot can be used as a spin entangler, ${ }^{12}$ with a spin singlet formed in dot A for an even number of electrons. As mentioned before, we can prepare an even number of electrons for each dot. The entangled spins are then separated and transferred to the two drain leads with one electron per path, thus creating spin entangled currents. References 13-15 predict the formation of a trapped state for electrons entering the triple dot via dots B and C. A coherent superposition of charge in the two dots can be created with destructive inter- ference at dot A blocking the transport. Depending on the needed direction of the current flow through the two paths, one could use different setups of source and drain contacts on our device to establish the appropriate conditions for both experiments.

In conclusion, we have investigated resonances of two and three quantum dots in transport and with charge detection in a three terminal lateral triple quantum dot. The three dots are arranged in a starlike geometry with each of them coupled to the other two. Three leads, one for each dot, allow for simultaneous transport measurements via different paths. Adjusting the four gate voltages, it was possible to establish resonances for all three dots. Via two paths, transport was simultaneously measured with each path showing resonances of two dots. The formation of quadruple points was simultaneously analyzed for both paths in the combined color scale plots for different electron numbers including one dot with only one or two electrons.

This work has been supported by BMBF via nanoQUIT. *rogge@nano.uni-hannover.de

${ }^{1}$ D. Loss and D. P. DiVincenzo, Phys. Rev. A 57, 120 (1998).

${ }^{2}$ L. P. Kouwenhoven, C. M. Marcus, P. L. McEuen, S. Tarucha, R. M. Westervelt, and N. S. Wingreen, in Mesoscopic Electron Transport, NATO Advanced Studies Institute, Series E: Applied Sciences, edited by L. L. Sohn, L. P. Kouwenhoven, and G. Schön (Kluwer, Dordrecht, 1997), Vol. 345, pp. 105-214.

${ }^{3}$ W. G. van der Wiel, S. De Franceschi, J. M. Elzerman, T. Fujisawa, S. Tarucha, and L. P. Kouwenhoven, Rev. Mod. Phys. 75, 1 (2003).

${ }^{4}$ A. W. Holleitner, C. R. Decker, H. Qin, K. Eberl, and R. H. Blick, Phys. Rev. Lett. 87, 256802 (2001).

${ }^{5}$ M. C. Rogge, C. Fühner, U. F. Keyser, R. J. Haug, M. Bichler, G. Abstreiter, and W. Wegscheider, Appl. Phys. Lett. 83, 1163 (2003).

${ }^{6}$ M. Pioro-Ladrière, M. Ciorga, J. Lapointe, P. Zawadzki, M. Korkusiński, P. Hawrylak, and A. S. Sachrajda, Phys. Rev. Lett. 91, 026803 (2003).

${ }^{7}$ J. M. Elzerman, R. Hanson, J. S. Greidanus, L. H. Willems van Beveren, S. De Franceschi, L. M. K. Vandersypen, S. Tarucha, and L. P. Kouwenhoven, Phys. Rev. B 67, 161308(R) (2003).

${ }^{8}$ J. R. Petta, A. C. Johnson, C. M. Marcus, M. P. Hanson, and A. C. Gossard, Phys. Rev. Lett. 93, 186802 (2004).

${ }^{9}$ A. K. Hüttel, S. Ludwig, H. Lorenz, K. Eberl, and J. P. Kotthaus, Phys. Rev. B 72, 081310(R) (2005).

${ }^{10}$ M. Field, C. G. Smith, M. Pepper, D. A. Ritchie, J. E. F. Frost, G. A. C. Jones, and D. G. Hasko, Phys. Rev. Lett. 70, 1311 (1993).
${ }^{11}$ M. C. Rogge, B. Harke, C. Fricke, F. Hohls, M. Reinwald, W. Wegscheider, and R. J. Haug, Phys. Rev. B 72, 233402 (2005).

${ }^{12}$ D. S. Saraga and D. Loss, Phys. Rev. Lett. 90, 166803 (2003).

${ }^{13}$ B. Michaelis, C. Emary, and C. W. J. Beenakker, Europhys. Lett. 73, 677 (2006).

${ }^{14}$ C. W. Groth, B. Michaelis, and C. W. J. Beenakker, Phys. Rev. B 74, 125315 (2006).

${ }^{15}$ C. Emary, Phys. Rev. B 76, 245319 (2007).

${ }^{16}$ R. J. Haug, Electrochim. Acta 40, 1283 (1995).

${ }^{17}$ F. R. Waugh, M. J. Berry, D. J. Mar, R. M. Westervelt, K. L. Campman, and A. C. Gossard, Phys. Rev. Lett. 75, 705 (1995).

${ }^{18}$ A. Vidan, R. M. Westervelt, M. Stopa, M. Hanson, and A. C. Gossard, Appl. Phys. Lett. 85, 3602 (2004).

${ }^{19}$ L. Gaudreau, S. A. Studenikin, A. S. Sachrajda, P. Zawadzki, A. Kam, J. Lapointe, M. Korkusinski, and P. Hawrylak, Phys. Rev. Lett. 97, 036807 (2006).

${ }^{20}$ D. Schröer, A. D. Greentree, L. Gaudreau, K. Eberl, L. C. L. Hollenberg, J. P. Kotthaus, and S. Ludwig, Phys. Rev. B 76, 075306 (2007).

${ }^{21}$ M. Ishii and K. Matsumoto, Jpn. J. Appl. Phys., Part 1 34, 1329 (1995).

${ }^{22}$ U. F. Keyser, H. W. Schumacher, U. Zeitler, R. J. Haug, and K. Eberl, Appl. Phys. Lett. 76, 457 (2000).

${ }^{23}$ With source at dot $\mathrm{B}$ and drain at $\mathrm{A}$ and $\mathrm{C}$, we found that the barrier connecting dots $\mathrm{B}$ and $\mathrm{C}$ cannot be opened while keeping the dots working even with $210 \mathrm{mV}$ applied to gate $G_{5}$. The low tunability originates from the fact that there is only one large gate $\left(G_{5}\right)$ on that side of the device. 